\title{
Recovery in Mental Health-A Community Based Wellness Group Model
}

\begin{abstract}
This paper presents a case study of a community-based intervention that supports older persons with mental illness. It also explains how the intervention minimises relapse, protracted hospital stays and repeat admissions for consumers with mental health problems who are at moderate to high risk factors. This paper proceeds on the basis of the WHO definition of mental health as "a state of wellbeing in which every individual realizes his or her own potential, can cope with the normal stresses of life, can work productively and fruitfully, and is able to make a contribution to her or his community" [1]. The community-based intervention titled the 'Wellness Group Program' is a holistic therapeutic program to assist older consumers with menta health problems in their recovery journey and to keep them well within the community.

Since its inception in 2001, there have been approximately 750 Wellness Group Program sessions within the Western Sydney Health District in the Australian state of New South Wales (NSW). During these 17 years, it provided therapeutic interventions for 12-18 consumers in each session and acted as a bridge between discharge from a hospital setting and community reintegration. Comparing relapse data and average length of stay for group participants with non-participants, the Wellness Group Program has been instrumental in freeing inpatient acute beds which has led to financial benefits for the health district. As such, it acted as a preventative vehicle and minimised relapse in patient's recovery journey in the community.
\end{abstract}

\section{The Context}

Changing demographics of older adults coupled with limited capacity in the hospital setting mandates a new way of managing the elderly consumers with mental health problems, in their recovery journey. As the stigma attached to mental health is addressed, with varying degrees of success, we are beginning to understand the global prevalence of mental illnesses. One in four people in the world will be affected by mental or neurological illnesses at some point in their lives (as indicated in WHO Health Report 2010) [2]. Worldwide, around 450 million people currently suffer from such conditions, placing mental disorders among the leading cause of ill-health and disability. On an average, there is only one psychiatrist per 100,000 people in over half the countries in the world. $40 \%$ of countries have less than one hospital bed reserved for mental disorders per 10,000 people [2]. In particular; loneliness, isolation and a lack of social connectedness have been found to be the major issues experienced by older population group hindering their mental health recovery.

Combined with this growing understanding of the prevalence of mental illness, is a demographic shift towards older persons in many parts of the world. The state of NSW, in Australia, where this experiment was undertaken, is already facing the dual challenges of increasing diagnoses of mental illness and a growing, aging population. The global population of older persons is projected to double in size from 903 million to 1.4 billion (UN Report). The number of people aged 80 years or over, the "oldest-old" persons,

\section{Journal of}

\section{Emergency Medicine \& Critical Care}

\section{Suman Tyagi* and Melinda Adamcewicz}

Older People's Mental Health Service, Western Sydney Local Health District, NSW Health, Australia

\section{Address for Correspondence}

Suman Tyagi, Older People's Mental Health Service, Western Sydney Local Health District, NSW Health, Australia, Tel: +61403113542; Email: suman.tyagi@health.nsw.gov.au

Submission: 20 May, 2018

Accepted: 18 June, 2018

Published: 25 June, 2018

Copyright: ๑ 2018 Tyagi S. et al. This is an open access article distributed under the Creative Commons Attribution License, which permits unrestricted use, distribution, and reproduction in any medium, provided the original work is properly cited.

is growing even faster than the number of older persons overall. In line with the global projected growth trend, the 65 years and older demographic is expected to experience the highest level of growth by 2031 in NSW (Graph 1) [3].

There are 240 beds in the Older People's Mental Health (OPMH) acute inpatient units in New South Wales, the most populous state in Australia, catering for more than one million elderly people. This equates to approximately 24 beds per 100,000 people. Unsurprisingly, Australia is already experiencing pressure on acute inpatient units due to the higher risk factors associated with the population of people aged 65 year and over, including biopsychosocial complexities and co-morbidities.

In light of a trend towards aging populations in several parts of the world, and noting the limitations of hospital-based recovery, the WHO Report in 2001 recommended that countries should move towards setting up community care alternatives, as traditional hospital type institutions are being phased out $[4,5]$. An important

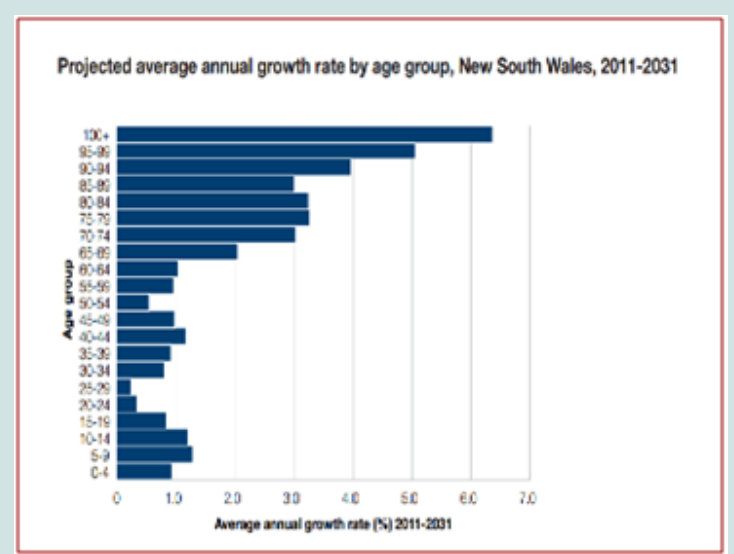

Graph 1: Projected annual growth rate by age group, NSW, 2011-2031 this demographic shift accompanies resource challenges to deal with the needs of this growing population of older persons. 
consideration for the above recommendation is the rate of relapse of affective disorders. These are disorders, such as depression and anxiety that have higher relapse rates, placing extra pressure on bed availability and acute services. Readmissions to Older Persons Mental Health (OPMH) acute inpatient units comprise $50 \%$ of repeat consumers within a 5-8 year period. However, studies have revealed that readmissions were less likely in a service that had a community orientation and where outpatient and community psychiatric followups were arranged [5].This framework for holistic treatment outside of the institutional inpatient setting can be visualized as a pyramid (Figure 1) [6]. The approach uses three parameters to build a coordinated response to mental health needs: the quantum of services needed; the frequency of needs; and the costs associated with each response.

\section{Supporting Recovery in the Community-'Right Care at the Right} Time'

The demographic shifts towards an aging population and successes in teaming inpatient treatment with community programs have led to the WHO recommending an approach whereby investment in mental health hospitals is capped and complemented by investment in other levels of the pyramid. Organising services and support in the community to assist with recovery is thus viewed as a good investment to increase access, treat mental health issues holistically and to reduce stigma [7].

In the NSW state of Australia, mental health funding has been consistently increasing to the community mental health sector. However, funding to psychiatric hospitals has reduced more than three-fold in last 30 years [8]. This reduction reflects an ongoing emphasis on using social support measures to maintain the recovery of consumers with mental health problems in a community setting. The healthcare initiatives of NSW Health Australia for 2013-14 advocate for the 'Right care in the right place at the right time in a rapidly changing environment' [9].

\section{The Conceptual Approach to the Wellness Group model}

This paper defines recovery to be a lived or real life experience

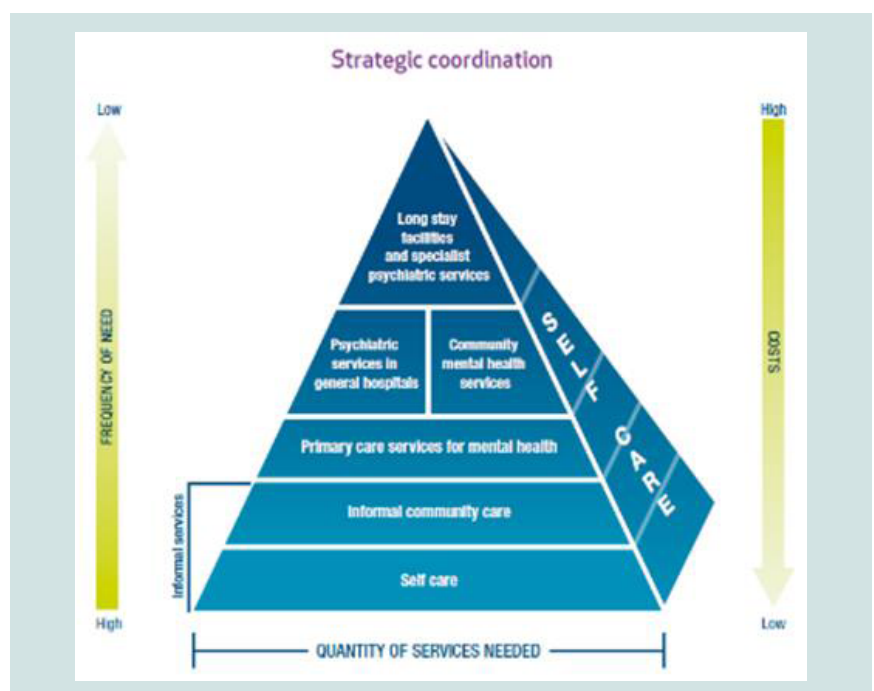

Figure 1: WHO Service Pyramid explaining hierarchy of services and their relationship with the frequency of services need and the financial impact [7]. of persons as they accept and overcome the challenge of an illness [10]. In this context, 'psychiatric rehabilitation' is the process aimed at enabling people who experience disabilities to reach and maintain their optimal physical, spiritual, occupational, sensory, intellectual, psychological and social functional levels [11]. This framework can also be used to characterise the inverse of an illness-the recovery.

The Wellness Group model is based on the understanding that recovery in mental health is achieved by a spectrum of interventions. It supports the participants at various stages/levels by way of establishing partnership and communication; assuring their rights, dignity and respect; recognising the uniqueness of the individual; exploring real choices/alternatives with them; and assisting, empowering, and enabling the person [12]. In-hospital treatment is only a component of the overall recovery process. The key is to support recovery outside the hospital, recognising that recovery is an ongoing process and that it needs a coordinated mental health care provision. These provisions need to be driven by a partnership approach and community-based recovery focused programs. This approach calls for a focus on individual strengths, interests, abilities and competencies, not deficits or pathology [2].

\section{A Community Based Wellness Group Model-Inception, Techniques and Logistics}

The inception of the community-based Wellness Group Program in 2001, was conceived as part of a national mental health strategy [13]. These strategies sought to address ongoing bed shortages and high re-admission rates by effectively supporting consumers' recovery outside the hospital setting through the provision of the communitybased interventions.

The Wellness Group Program was a holistic therapeutic program started by clinicians working across the inpatient and community setting. It helped clinicians to provide continuity of care and positive outcomes. It was based on the understanding that the communitybased programs were easily accessible, hence likely to be the most acceptable, effective and least stigmatising. It acknowledged the importance of recovery language and that the language needs to be respectful, non-judgmental, carry a sense of commitment, hope and present the potential for opportunity [14]. The overall aim was to improve outcomes in patients within the mental health system and achieve greater efficiencies of care while providing a better experience for consumers. The Wellness Group Program is designed to provide specialist mental health services to older consumers who present with higher risks due to their complex bio-psychosocial contexts/ circumstances. It aimed to assist with consumer awareness of mental and physical health problems, as evident from the wide range of topics covered in the group.

As a result, the Wellness Group Program has increased consumers' understanding of early warning signs and treatment options. It has assisted them with the management of mental health problems, coping strategies and overall wellbeing care plans. The program facilitates formal and informal interactions between consumers in an environment where consumers can share experiences and learn about managing their mental and general wellbeing.

The overall effect is to improve the throughput of patients within the mental health system and achieve greater efficiencies of care while providing a better experience for consumers. 
The program design: The Wellness Group Program is designed to provide specialist mental health services to older consumers who present with higher risks due to their complex bio-psychosocial contexts/circumstances. It aimed to assist with consumer awareness of mental and physical health problems through customised program contents and a wide range of topics in providing a comprehensive approach.

Eligibility to the group: The program aimed to provide specialist mental health services to older consumers who present with higher risks due to their complex bio-psychosocial contexts/circumstances. The Wellness Group is open to all who present with mental health issues and are suitable for recovery and psychiatric rehabilitation. The program is suited for those who have the motivation and willingness to attend the group, reasonable English skills and largely intact cognition and memory.

Referrals to the program: Referral for the Wellness Group Program can be made at the same central intake point (statewide mental health line) as for community based mental health interventions and case management. Referrals to this communitybased wellness program can also be made from a range of public or private sources including hospitals, Community Health Services, and General Practitioners.

The referral form is specifically designed for this program. Following a referral to the service, the program coordinators assess case needs and appropriateness of each referred patient to attend the group.

Documentation: The members of the Wellness Group Program are registered on an electronic medical record (EMR- Cerner Millennium- Power Charts). This includes frequency of the group, length of treatment, and a list of patients. A record of participation in the group is maintained to follow up progress and provide appropriate interventions. Consumers' attendance and participation in the group is recorded in electronic medical records. The individual record also contains mental health activities, including the time spent on each activity by the clinicians facilitating the group.

Mental health reviews: Mental health reviews are arranged for the participants as per NSW health clinical guidelines (at least once in three months) or according to their clinical needs. Mental Health Outcome and Assessment Tools (MH OAT) including Standard Measures and K-10 (Self-reporting measure Kessler-10), are completed as per mental health state-wide guidelines and clinical needs [15]. The consumer and carers are involved in the development of the mental health care plan. A nation-wide feedback survey called YES (Your experience of Service) is given to the consumers as part of the NSW health guidelines. Additionally, an evaluation and feedback survey, specially designed for the program, is conducted on a regular basis with consumers to incorporate their opinions on various aspects of the conduct and contents of the program. The design of the Wellness Group Program is dynamic and flexible that responds to feedback by consumers.

Arrival and waiting area: Consumers notify at the reception of the local community health centre and get a visitor's badge. Consumers arrive and gather in the waiting area before the group starts. Pre-group get together acts as a platform to explore mutual support and socialisation possibilities. This comradery gives group members an opportunity for sharing their emotions in a safe and non judgmental environment.

Conduct of the group: Up to 18 consumers attend a weekly semiclosed community group session led by the senior social worker and an occupational therapist.

Wellness group general session plan: This includes welcome to the group with afternoon tea while soothing music is played. This is followed by an informal catch-up with the participants followed by every participant reading a motivational and positive quote from the healing cards and booklets. The group members have contributed to this collection that started only with one pack of healing cards. This involvement and ability to add their contribution gives them a sense of empowerment and ownership. It encourages the participants to selfreflect and share their experiences. Gentle Exercise for ten minutes to encourage the regular practice and to remind them of its benefits is the next step of the program which is followed by the 'topic of the day'. There are range of topics related to mental and physical wellbeing included in the program Consumers's feedback and suggestions are considered valuable while designing the program, session plan and the topics. The group ends with a Progressive Muscle Relaxation exercise with music playing in the background. The group members leave after a brief Cool-Down period.

Outcomes and evaluation: The community-based Wellness Group Recovery Model is both delivery and capability oriented (Figure 2). It not only delivers the outcomes but also builds the capacity of the system to assist consumers outside the hospital setting. It supports consumers, carers as well as mental health professionals.

Service provision: Since 2001, there have been approximately 750 weekly sessions (44 weeks per year approx.) providing recovery oriented therapeutic interventions to an average of 12 consumers in each Wellness Group Program at the community setting, totaling at least 9000 occasions of mental health activity for these consumers since its inception.

Opportunities for replication or scaling up: The Wellness Group Program has been adopted by other health districts of NSW Health. The program is aligned with the current Community Model of Care Guideline, Acute Inpatient Model of Care Guidelines,

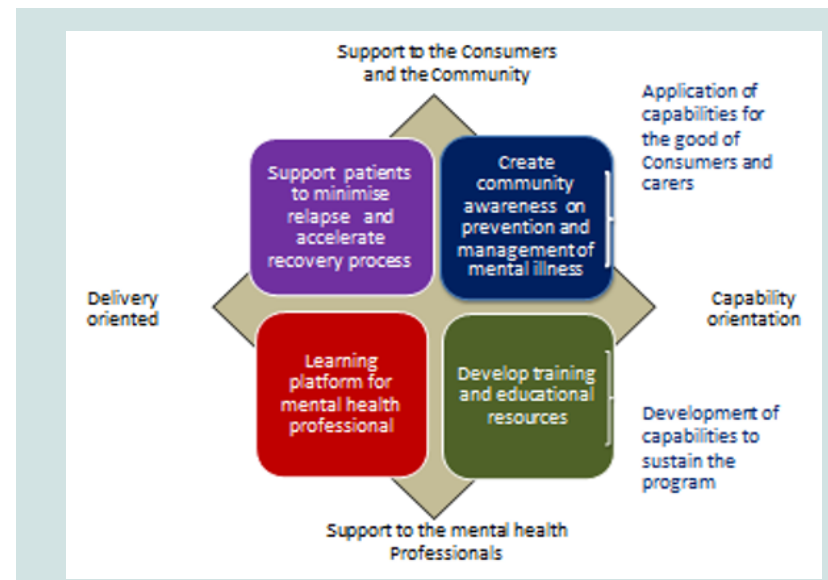

Figure 2: Wellness group recovery model. 
Specialist Mental Health Service for Older People and Recoveryoriented Practice Improvement Project, NSW Older People's Mental Health [16-18].

Financial impact: It is a low-cost program as it uses existing clinicians working across inpatient and community setting. Light refreshments served to group members cost less than $\$ 500$ per annum. The booklets provided to consumers are printed, copied and bounded for an approximate cost of $\$ 100$ annually.

The program assists with timely discharge from an acute inpatient setting by providing continuity of therapeutic interventions through this program, leading to freeing inpatient beds. The Program has saved acute bed days, amounting to a saving in bed costs for the health district.

The program has promoted consumer recovery process by assisting them with community reintegration and social connectedness. It provides early interventions and thus reduces relapse rate amongst the program members requiring re-admission in the hospital. Despite complex and high-risk profile of participants, the readmission rate of these patients has been consistently below the state average.

\section{References}

1. WHO, The Calouste Gulbenkian Foundation (2014) Social determinants of mental health. World Health Organisation, Portugal, pp. 1-54.

2. WHO (2010) Rehabilitation, health topics. World Health Organisation. Geneva, Switzerland.

3. (2009) National survey of mental health and wellbeing: summary of results 2007. Australian Bureau of Statistics.
4. (2012) Specialist Mental Health for Older People (SMHSOP) model of care report NSW Health, Australia, pp. 1-104.

5. WHO (2001) The world health report 2001-Mental health: new understanding, new hope. World Health Organisation, Geneva, Switzerland, pp. 1-104.

6. (2007) The WHO mind project, mental improvement for nations development. World Health Organisation, Geneva, Switzerland, pp. 1-3.

7. Whiteford HA, Buckingham WJ (2005) Ten years of mental health service reform in Australia: are we getting it right? Med J Aust 182: 396-400.

8. (2013) Department of Health and Ageing (DoHA) - Medicare Australia Memorandum of Understanding (MoU). pp. 1-48.

9. (2013) 2013-14 Key budget facts NSW health government, Australia, pp.1-14.

10. Deegan P (1988) Recovery: the lived experience of rehabilitation. Int $J$ Psychosoc Rehabil 11: 11.

11. (2004) A recovery vision of rehabilitation psychiatric rehabilitation policy and strategic framework. Department of Health, Australia, pp. 1-68.

12. (2010) Collaborative care model National Advisory Council on Mental Health Australian 2010.

13. (2001) International mid-term review of the second national mental health plan of Australia. The Department of Health, Australia.

14. (2018) Recovery oriented language guide, mental health coordinating council NSW Health, Australia, pp 1-12.

15. (2014) Mental health clinical documentation guidelines. Ministry of Health, NSW, Australia, pp. 1-28.

16. (2017) Specialist Mental Health Services for Older People (SMHSOP) community model of care guideline, NSW Health, Australia, pp. 1-119.

17. (2016) NSW SMHSOP acute inpatient unit model of care guideline. NSW Health, Australia, pp.1-47.

18. (2018) Recovery-oriented Practice Improvement Project, NSW older people's mental health, NSW Ministry of Health, Australia, pp. 1-91. 